\title{
Uniqueness of the Solutions of Sigma Models in Non-Riemannian Background
}

\author{
METIN GÜRSES \\ Department of Mathematics, Faculty of Science, Bilkent University, 06533 Ankara, Turkey \\ c-mail: gurses $(a$ trbilun \\ (Received: 28 August. 1992)
}

\begin{abstract}
It is proved that the boundary value problems of some sigma-models in a non-Riemannian background have unique solutions. Sigma models on Riemannian backgrounds, sigma models with a Wess-Zumino-Witten term, the Ward model, and the self-dual Yang-Mills equations are among these models.
\end{abstract}

Mathematics Subject Classifications (1991). 8IE13, 81E20, 35J60, $35 \mathrm{~J} 65$.

In a recent paper [1], we have investigated the classical integrability of the sigma models in a non-Riemannian background and have given their one-soliton Backlund transformations. In particular, two-dimensional sigma-models with a WessZumino Witten term have been studied in detail.

Let $M$ be an $m$-dimensional manifold with the local coordinates $x^{\mu}$ and $\Lambda^{\mu v}$ components of a complex tensor field in $M$. Let $P$ be an $n \times n$ matrix with $\operatorname{det}(P)=1$. We also assume that $P$ is a Hermitian $\left(P^{\dagger}=P\right)$ matrix. Then the sigma-model we consider is given as

$$
\frac{\partial}{\partial x^{\alpha}}\left(\Lambda^{\alpha \beta} P^{-1} \frac{\partial P}{\partial x^{\beta}}\right)=0 \text {. }
$$

For various choices of the tensor field $\Lambda$, the above equation arises in several branches of mathematical physics: (i) $m=2$ and $\Lambda^{\alpha \beta}$ is real and symmetric. Equation (1) represents the essential part of the stationary axially symmetric Einstein- $n$-Maxwell field equations [5-8]. In this case,

$$
P \varepsilon \frac{\mathrm{SU}(n+1,1)}{\mathrm{SU}(n+1) \times \mathrm{U}(1)} .
$$

Uniqueness of these solutions, in particular the uniqueness of the rotating charged black hole solution, has been shown by [2] and [4]. This proof and its extensions have been studied in detail in [10] and [11]. (ii) $m=2$ and $\Lambda^{\alpha \beta}$ is real, symmetric, and constant. Equation (1) represents the sigma model which is used as a nonperturbative field theory. (iii) $m=2, \Lambda^{\alpha \beta}$ is real, constant, and also carries an antisymmetric part. This is the sigma model with a Wess-Zumino-Witten (WZW) term. 
(iv) $m=3, \Lambda^{\alpha \beta}$ is real with constant entries. This is the Ward model [9]. (v) $m=4$ and $\operatorname{Det}\left(\Lambda^{\alpha \beta}\right)=0$. Equation (1) represents the self-dual Yang-Mills equations in Yang`s $R$-gauge.

In [1], we have shown that $\Lambda^{\alpha \beta}$ has to satisfy some algebraic constraints for $m>2$ and differential constraints for $m=2$ for an integrable subclass of (1). Solutions of these constraints indicate that there are also nonsymmetric tensor fields $\Lambda^{\alpha \beta}$ for which Equation (1) is integrable. Some specific examples of this class are signa models with a WZW term when $m=2$ and the self-dual Yang-Mills equations when $m=4$. Uniqueness theorems of [2-4] are not applicable to this class. In their treatment, they assume a Riemannian background (i.e., real and symmetric $\Lambda^{\alpha \beta}$ ).

The purpose of this Letter is to search for a class of sigma models which has unique solutions. We show that this class is quite large and contains the models on Riemannian backgrounds, sigma models with a WZW term, the Ward model, and the self-dual Yang-Mills equations.

In the sequel, we assume enough differentiability for the components of the matrix $P$ and the tensor field $\Lambda^{\alpha \beta}$ in $D \cup \partial D$ and follow the method of [4] in a slightly different way. Here $D$ is a region in $M$ with boundary $\partial D$. We also assume that $P$ is positive definite. Let $P_{1}$ and $P_{2}$ be two different solutions of (1). The difference of their equations satisfy

$$
\frac{\partial}{\partial x^{\alpha}}\left(\Lambda^{\alpha \beta} P_{1}^{-1} \frac{\partial Q}{\partial x^{\beta}} P_{2}\right)=0
$$

where $Q=P_{1} P_{2}^{-1}$. Multiplying both sides by $Q^{\dagger}$ (Hermitian conjugation) and taking the trace, we obtain

$$
\frac{\partial}{\partial x^{\alpha}}\left[\Lambda^{\alpha \beta} \operatorname{tr}\left(Q^{\dagger} P_{1}^{-1} \frac{\partial Q^{\dagger}}{\partial x^{\beta}} P_{2}\right)\right]=\operatorname{tr}\left[\Lambda^{\alpha \beta}\left(\frac{\partial Q^{\dagger}}{\partial x^{\alpha}}\right) P_{1}^{-1}\left(\frac{\partial Q}{\partial x^{\beta}}\right) P_{2}\right]
$$

The left-hand side of the above equation can be simplified further and we obtain

$$
\frac{\partial}{\partial x^{\alpha}}\left(\Lambda^{\alpha \beta} \frac{\partial q}{\partial x^{\beta}}\right)=\operatorname{tr}\left[\Lambda^{\alpha \beta}\left(\frac{\partial}{\partial x^{\alpha}} Q^{\dagger}\right) P_{1}^{-1}\left(\frac{\partial Q}{\partial x^{\beta}}\right) P_{2}\right],
$$

where $q=\operatorname{tr}(Q)$. Using the hermiticity and positive definiteness properties of the matrices $P_{1}$ and $P_{2}$, we may let

$$
P_{i}=A_{i} A_{i}^{\dagger} \quad(i=1,2)
$$

where $A_{1}$ and $A_{2}$ are nonsingular $n \times n$ matrices. With the aid of (5), Equation (4) reduces to

$$
\frac{\partial}{d x^{\alpha}}\left(\Lambda^{\alpha \beta} \frac{\partial q}{\partial x^{\beta}}\right)=\operatorname{tr}\left(\Lambda^{\alpha \beta} J_{\alpha}^{\dagger} J_{\beta}\right)
$$

where

$$
J_{\alpha}=A_{1}{ }^{\prime}\left(\frac{\partial Q}{\partial x^{\alpha}}\right) A_{2}
$$


Equation (6) is a crucial step towards the proof of the uniqueness theorems. Before going on, let us give an example for the scalar function $q$. It is positive definite for all $n$. As an illustration for $n=2$, the matrix $P$ takes the form

$$
P=\left(\begin{array}{cc}
\rho & \omega \\
\omega^{*} & \frac{1+\omega \omega^{*}}{\rho}
\end{array}\right)
$$

where $\rho$ is real (and positive in $D$ ), $\omega$ are complex functions, and $*$ denotes complex conjugation. We find that

$$
q=2+\frac{1}{\rho_{1} \rho_{2}}\left[\left(\rho_{1}-\rho_{2}\right)^{2}+\left|\rho_{2} \omega_{1}-\rho_{1} \omega_{2}\right|^{2}\right] .
$$

It is clear that $q=2$ on the boundary $\partial D$ of $D$. In general (for all $n$ ), $q$ takes the form

$$
q=n+\text { sum of positive terms. }
$$

If $q$ equals $n$, then each positive term in (9) must vanish. This implies $P_{1}=P_{2}$.

For our later purposes, it is better to separate (6) into its real and imaginary parts. Let $\Lambda_{\alpha \beta}=g^{\alpha \beta}+i h^{\alpha \beta}$. Here $g^{\alpha \beta}$ and $h^{\alpha \beta}$ are, respectively, the real and imaginary parts of the tensor $\Lambda^{\alpha \beta}$. Recalling that $a^{\alpha \beta} \operatorname{tr}\left(J_{\alpha}^{\dagger} J_{\beta}\right)$ is real for real symmetric tensor $a^{\alpha \beta}$ and $b^{\alpha \beta} \operatorname{tr}\left(J_{\alpha}^{\dagger} J_{\beta}\right)$ is pure imaginary for real and antisymmetric tensor $b^{x \beta}$, we find that

$$
\begin{aligned}
& 2 \frac{\partial}{\partial x^{\alpha}}\left(g^{\alpha \beta} \frac{\partial q}{\partial x^{\beta}}\right)=\left(g^{\alpha \beta}+g^{\beta \alpha}\right) \operatorname{tr}\left(J_{\alpha}^{\dagger} J_{\beta}\right)+i\left(h^{\alpha \beta}-h^{\beta \alpha}\right) \operatorname{tr}\left(J_{\alpha}^{\dagger} J_{\beta}\right), \\
& 2 \frac{\partial}{\partial x^{\alpha}}\left(h^{\alpha \beta} \frac{\partial q}{\partial x^{\beta}}\right)=-i\left(g^{\alpha \beta}-g^{\beta \alpha}\right) \operatorname{tr}\left(J_{\alpha}^{\dagger} J_{\beta}\right)+\left(h^{\alpha \beta}+h^{\beta \alpha}\right) \operatorname{tr}\left(J_{\alpha}^{\dagger} J_{\beta}\right) .
\end{aligned}
$$

Before giving the uniqueness proofs, let us first state the conditions that we need in these proofs.

\section{Uniqueness Conditions (UC)}

Let $M$ be an $m$-dimensional manifold with local coordinates $x^{x}$. Let $D$ be a region in $M$ with boundary $\partial D$. Let $\Lambda^{\alpha \beta}$ be the components of a complex tensor field in $D$ with enough differentiability conditions. Let $P$ be a Hermitian positive definite $n \times n$ matrix with unit determinant and let $P_{1}$ and $P_{2}$ be two such matrices satisfying (1) in $D$ with the same boundary conditions on $\partial D$, then we have

LEMMA 1. $P_{1}=P_{2}$ at all points in region $D$ if $g^{\alpha \beta}+g^{\beta \alpha}$ is positive definite, nondegenerate, and $h^{\alpha \beta}=h^{\beta \alpha}$. 
Proof. Integrating (10) in $D$ we obtain

$$
\int_{\hat{C} D} g^{\alpha \beta} \frac{\partial q}{\partial x^{\beta}} \mathrm{d} \Sigma_{\alpha}=\frac{1}{2} \int D\left(g^{\alpha \beta}+g^{\beta \alpha}\right) \operatorname{tr}\left(J_{\alpha}^{\dagger} J_{\beta}\right) \mathrm{d} V
$$

and, using the boundary condition $q=n$ on $\partial D$, we get

$$
\int_{D}\left(g^{\alpha \beta}+g^{\beta \alpha}\right) \operatorname{tr}\left(J_{\alpha}^{\dagger} J_{\beta}\right) \mathrm{d} V
$$

Since the symmetric part of $g^{\alpha \beta}$ is positive definite, then the integrand in (13) vanishes at all points in $D$. This and the nondegeneracy of $g^{\alpha \beta}+g^{\beta \alpha}$ imply the vanishing of $J_{x}$ which implies that $Q=Q_{0}=$ a constant matrix in $D$. Since $Q$ is the identity matrix $I$ on $\partial D$, then $Q=I$ in $D$. Hence, $P_{1}=P_{2}$ at all points in $D$. Another way to prove this lemma is to use (10) directly. The vanishing of the integrand in (10) implies that $q$ is an harmonic function in $D$ with respect to a nondgenerate and positive definite metric $g^{\alpha \beta}+g^{\beta x}$. Since $q=n$ on the boundary $\delta D$ of $D$, then it must be equal to the same constant in $D$ as well. This implies that $P_{1}=P_{2}$ in $D$.

COROLLARY. Sigma models with $h^{\alpha \beta}=0$ such as sigma models on Riemannian geometries, sigma models with a Wess-Zumino Witten term $(m=2)$, and the Ward model $(m=3)$ have all unique solutions provided the conditions of the above lemma are satisfied.

In the proof of the above lemma, we have not used Equation (11). We remark that there is a duality in Equations (10) and (11). This property enables us to state a new lemma.

LEMMA 2. Lemma 1 and its proof remain valid if $g^{\alpha \beta}$ and $h^{\alpha \beta}$ are interchanged.

The imaginary part of $\Lambda^{\alpha \beta}$ in self-dual Yang-Mills equations is antisymmetric. Hence, the above lemma cannot be utilized for these type of equations. Hence, we have the following lemma.

LEMMA 3. $P_{1}=P_{2}$ at all points in region $D$ if $\Lambda^{\alpha \beta}=\Sigma_{k=1}^{r} u_{k}^{\alpha \dagger} u_{k}^{\beta}$, where $u_{k}^{\alpha}$ are complex vectors in $M$. Here, $r=m / 2$ when $m$ is even and $r=(m-1) / 2+1$ when $m$ is odd and one of the vectors $u_{k}^{x}$ is real.

Proof. Defining $j_{k}=u_{k}^{\alpha} J_{\alpha}$ and using the boundary conditions in Equation (6), we obtain

$$
\int_{D} \sum_{k=1}^{r} j_{k}^{\dagger} j_{k} \mathrm{~d} V=0
$$

Since the integrand is positive definite, then it must vanish. Together with (6), $q$ is an harmonic function in $D$ with respect to the metric $\Lambda^{\alpha \beta}+\Lambda^{\beta \alpha}$ which is positive definite and nondegenerate. Positive definiteness follows from $\Lambda^{\alpha \beta}=\Sigma_{k=1}^{r} u_{k}^{\alpha \dagger} u_{k}^{\beta}$. Letting $u_{k}^{\alpha}=a_{k}^{\alpha}+i b_{k}^{\alpha}$, where $a_{k}^{\alpha}$ and $b_{k}^{\alpha}$ are real vectors,

$$
\Lambda^{\alpha \beta}=\sum_{k=1}^{r}\left(a_{k}^{\alpha} a_{k}^{\beta}+b_{k}^{\alpha} b_{k}^{\beta}\right)+i \sum_{k=1}^{r}\left(a_{k}^{\alpha} b_{k}^{\beta}-a_{k}^{\beta} b_{k}^{\beta}\right) \text {. }
$$


When $m=$ odd integer, one of the vetors $b_{k}^{\alpha}$ is zero. The real and symmetric part of $\Lambda^{x f}$ is written in terms of $m$ distinct real vectors, hence it is also nondegenerate.

COROLLARY. The self-dual Yang-Mills equations have unique solutions. Here $m=4$ and

$$
a_{1}^{\alpha}=(1,0,0,0), \quad b_{1}^{\alpha}=(0,1,0,0), \quad a_{2}^{\alpha}=(0,0,1,0), \quad b_{2}^{\alpha}=(0,0,0,1) .
$$

\section{Acknowledgement}

This work is partially supported under TBAG/CG-1 by the Scientific and Technical Research Council of Turkey (TUBITAK).

\section{References}

1. Gürses, M. and Karasu, A., Internat. J. Modern Phys. A 6, 487 (1991).

2. Bunting, G., The black hole uniqueness theorem, in Proc. Australlan Mathematics Convention, Sydney, 1981.

3. Bunting, G.. Proof of the uniqueness conjecture for black holes, PhD thesis, University of New England, Armidale, N.S.W., 1983.

4. Mazur, P. O., J Phys. A 15, 3173 (1982).

5. Mazur, P. O., Acta Phys. Polon. B 14, 219 (1983).

6. Gürses, M. and Xanthopoulos, B., Phys. Rev. D 26. 1912 (1982).

7. Gürses, M., Phys. Rev D 30, 486 (1984).

8. Eriş. A., Gürses, M., and Karasu, A., J. Math. Phys. 25, 1489 (1984).

9. Ward, R., J. Math. Phys. 29, 386 (1988).

10. Bretenlohner, P., Maison, D., and Gibbons, G., Four-dimensional black holes from Kaluza-Klein theories. Preprint MPI-PAE/PTh 27/87, LPTENS 87/9 (March 1987).

11. Carter, B., Comm. Math. Phys. 99. 563 (1985). 